\title{
Comprehensive oligonucleotide array-comparative genomic hybridization analysis: new insights into the molecular pathology of the DMD gene
}

\author{
Aliya Ishmukhametova ${ }^{\star, 1,2}$, Philippe Khau Van Kien ${ }^{3,7}$, Déborah Méchin ${ }^{3}$, Delphine Thorel ${ }^{3}$, \\ Marie-Claire Vincent ${ }^{3}$, François Rivier ${ }^{4,5}$, Christine Coubes ${ }^{6}$, Véronique Humbertclaude ${ }^{1,2}$, \\ Mireille Claustres ${ }^{1,2,3}$ and Sylvie Tuffery-Giraud ${ }^{\star, 1,2}$
}

We report on the effectiveness of a custom-designed oligonucleotide-based comparative genomic hybridization microarray (array-CGH) to interrogate copy number across the entire 2.2-Mb genomic region of the DMD gene and its applicability in diagnosis. The high-resolution array-CGH, we developed, successfully detected a series of $\mathbf{4 2}$ previously characterized large rearrangements of various size, localization and type (simple or complex deletions, duplications, triplications) and known intronic CNVs/Indels. Moreover, the technique succeeded in identifying a small duplication of only $191 \mathrm{bp}$ in one patient previously negative for $D M D$ mutation. Accurate intronic breakpoints localization by the technique enabled subsequent junction fragments identification by sequencing in $86 \%$ of cases (all deletion cases and $62.5 \%$ of duplication cases). Sequence examination of the junctions supports a role of microhomology-mediated processes in the occurrence of DMD large rearrangements. In addition, the precise knowledge of the sequence context at the breakpoints and analysis of the resulting consequences on maturation of pre-mRNA contribute to elucidating the cause of discrepancies in phenotype/genotype correlations in some patients. Thereby, the array-CGH proved to be a highly efficient and reliable diagnostic tool, and the new data it provides will have many potential implications in both, clinics and research.

European Journal of Human Genetics 20, 1096-1100; doi:10.1038/ejhg.2012.51; published online 18 April 2012

Keywords: duchenne muscular dystrophy; $D M D$ gene; large rearrangements; comparative genomic hybridization microarray; diagnostic methods

\section{INTRODUCTION}

Mutations in the huge human Duchenne muscular dystrophy gene (DMD; MIM\#300377), which encodes the 427-kDa muscular dystrophin protein isoform, result in dystrophinopathies. There is no simple relationship between the type or the size of the mutations in the $D M D$ gene and the severity of phenotype, but the reading-frame rule holds true for $96 \%$ of Duchenne Muscular Dystrophy (DMD; OMIM\#310200) and 93\% of Becker Muscular Dystrophy (BMD; OMIM\#300376) cases. $^{1}$ As the majority of mutations in DMD are large deletions and duplications, several dosage-sensitive quantitative methods mainly focused on discovering mutations in the coding regions of the gene are commonly used. ${ }^{2}$ Here we describe the introduction into the current diagnostic practice and validation of a high-resolution custom-designed Comparative Genomic Hybridization array (array-CGH) enabling to interrogate the entire $2.2 \mathrm{Mb}$ genomic region of the $D M D$ gene for copy number variations. A panel of $D M D$ rearrangements of various type, size and localization was selected, some of which did not conform to the reading-frame rule. Eight mutation-negative patients were also analyzed. We specifically assessed the ability of the custom-designed array-CGH to detect rearrangements within the $D M D$ gene and the potential contribution of this method to the identification of breakpoint/ junction sequences.

\section{MATERIALS AND METHODS \\ Patients}

Based on the data available in the clinical and molecular databases maintained in our laboratory, ${ }^{1}$ we selected 50 patients' DNA from previously collected 550 non-related DMD/BMD families (French Ministry of Health, collection ID: DC-2008-417) dividing them into three groups (Table 1; Supplementary Table 1). All patients provided an agreement for further analysis on informed consent form. Eight relatives from four unrelated families were included to test the reproducibility of the technique. DNA samples of Marfan patients (two males and one female) with previously identified large rearrangements in the fibrillin type 1 (FBN1) gene $\mathrm{g}^{3}$ and no familial history of neuromuscular disorders, served as gender-matched internal positive controls for array-CGH

\section{Array-CGH}

The Roche NimbleGen (Roche NimbleGen, Inc., Madison, WI, USA) customdesigned $12 \times 135 \mathrm{~K}$ format contained 3440 exonic DMD probes, overlapped

${ }^{1}$ Université Montpellier 1, UFR médecine, Montpellier, France; ${ }^{2}$ INSERM, U827, Montpellier, France; ${ }^{3} \mathrm{CHU}$ Montpellier, Hôpital Arnaud de Villeneuve, Laboratoire de Génétique Moléculaire, Montpellier, France; ${ }^{4} \mathrm{CHRU}$ de Montpellier, Neuropédiatrie CR Maladies Neuromusculaires, Montpellier, France; ${ }^{5}$ U1046, INSERM, Université Montpellier 1 et 2 , Montpellier, France; ${ }^{6} \mathrm{CHU}$ Montpellier, Hôpital Arnaud de Villeneuve, Service de Génétique Médicale, Montpellier, France

7Present address: $\mathrm{CHU}$ de Nîmes, Laboratoire de Cytologie Clinique et Cytogénétique, Nîmes, France.

*Correspondence: Dr A Ishmukhametova or Dr S Tuffery-Giraud, INSERM, U827, IURC, Institut Universitaire de Recherche Clinique, 641 Avenue du Doyen Giraud, 34093 Montpellier Cedex 5, France. Tel: +33 4117598 79; Fax: + 334117598 82; E-mail: aliya.ishmukhametova@inserm.fr or sylvie.tuffery@inserm.fr

Received 7 November 2011; revised 27 January 2012; accepted 21 February 2012; published online 18 April 2012 


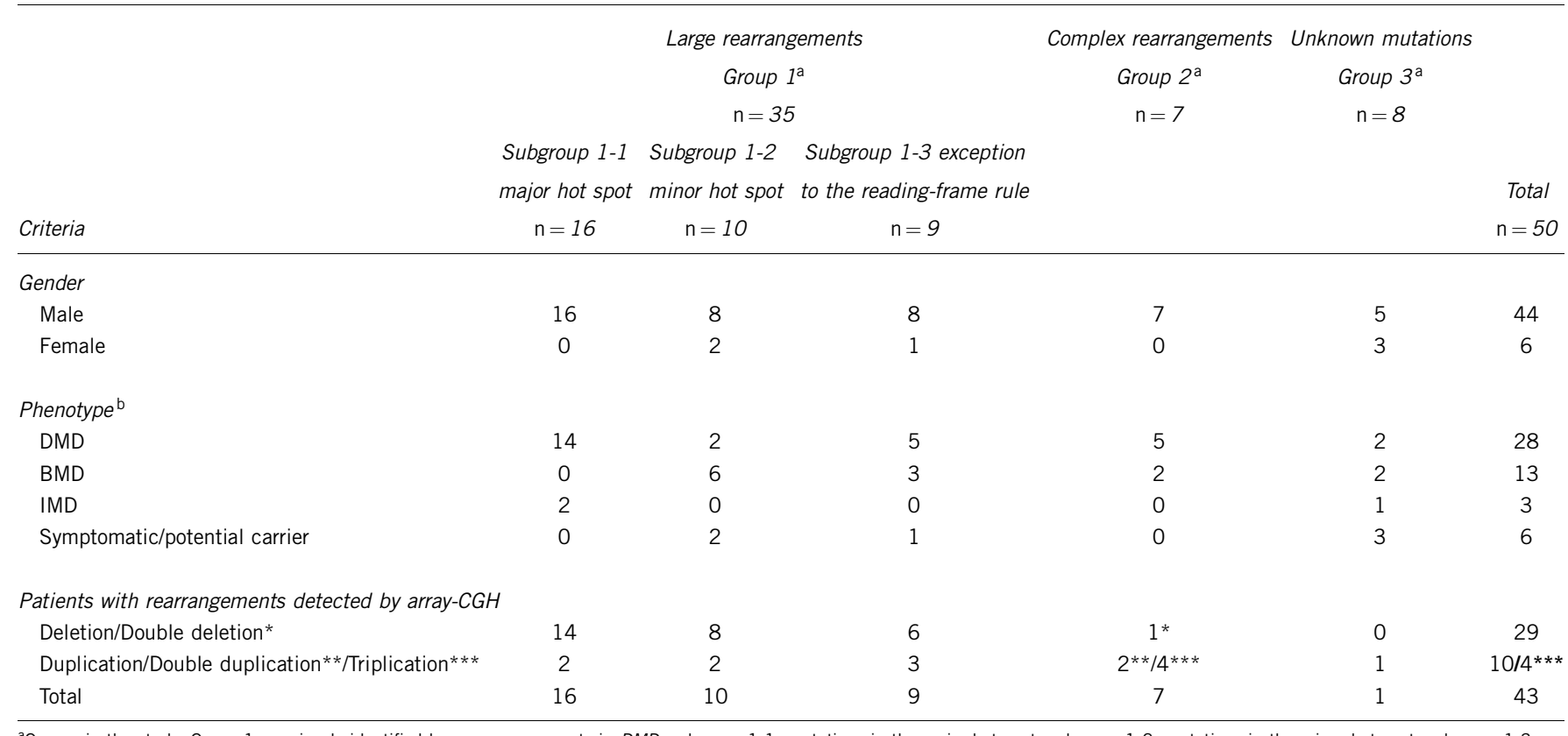

aGroups in the study: Group 1, previously identified large rearrangements in DMD: subgroup 1-1, mutations in the major hot spot; subgroup 1-2, mutations in the minor hot spot; subgroup 1-3, other exceptions to the reading-frame rule. Group 2, previously identified complex rearrangements, more than one alteration found in $D M D\left({ }^{*}\right.$ double deletion, ${ }^{* *}$ double duplication, ${ }^{* * *}$ triplication). Group 3, patients with undetected defect in DMD (by multiplex-PCR, semi-quantitative-PCR, Multiplex ligation-dependent probe amplification, whole-exon sequencing).

bPhenotype: DMD, Duchenne phenotype; BMD, Becker phenotype; IMD, intermediate phenotype; Symptomatic carrier, female with clinical symptoms of any severity; potential carrier, female with elevated creatine kinase levels.

and shifted on an average of 10 bases, and 19294 intronic DMD probes interspersed by $100 \mathrm{bp}$ on average. Slides were scanned by InnoScan $900 \mathrm{~A}$ (Inopsys, Toulouse, France) and analyzed using the CGH-segMNT algorithm of NimbleScan version 2.5 software (Roche NimbleGen, Inc.). The predicted breakpoint location was defined by the positions of the last and first probes with normal unaveraged value of $\log _{2}$-ratio upstream and downstream from the corresponding aberration.

\section{PCR/Sequencing across the breakpoints}

PCR primers were designed in an average distance of $0.7 \mathrm{~kb}$ upstream and downstream of each predicted junction and amplifications were performed using standard protocols of Promega Master Mix (Promega Corporation, Madison, WI, USA), Phusion Hot Start High-Fidelity DNA polymerase (Finnzymes Oy, Espoo, Finland) or LongRange PCR kit (Qiagen, Courtaboeuf, France). When obtained, amplified junction fragments were sequenced using the Big Dye terminator version 1.1 Cycle Sequencing Kit (Applied Biosystems, Courtaboeuf, France).

\section{Bioinformatic analysis}

UCSC Genome Browser (http://genome.ucsc.edu) and BLAST program (http://blast.ncbi.nlm.nih.gov/) were used for the mapping the particular motifs surrounding the junctions. The Position Converter Interface in Mutalyzer 2.0 $\beta-8$ was applied to convert chromosomal positions of Mar.2006 NCBI Build 36.1/hg18 (RefSeq NC_000023.9) to transcript orientated positions. ${ }^{4}$

\section{RESULTS}

\section{Array-CGH: sensitivity and reproducibility}

The array-CGH analysis confirmed all 35 large deletions and duplications and 7 complex contiguous and non-contiguous rearrangements (Figure 1) previously identified in patients from group 1 and 2, giving a $100 \%$ detection rate (Table 1, Supplementary Table 2). The method was also able to detect a hemizygous 191-bp duplication spanning intron 19-exon 20 junction in one DMD patient (D87, group 3), which had escaped detection both by MLPA (due to a probe-target mismatch at the $3^{\prime}$ end of the MLPA probe) and by genomic sequencing (due to the parameter settings for the sequencing analysis software used)

Independently derived data from eight tested relatives from four different families and from duplicate experiments performed for 12 patients showed that the reproducibility of our array-CGH platform was high, with an average accuracy in the breakpoint localization of about $700 \mathrm{bp}$ (range 0-4 kb) (Supplementary Table 2). Apart from the large rearrangements already known and correctly predicted by arrayCGH, we noticed some experimental artefacts (ie data not confirmed on independent and/or averaged results of array-CGH) in the vicinity of exons 13, 17, 45, Dp140, Dp71 and intron 67.

\section{Sequence characteristics at the breakpoints}

The accuracy of array-CGH breakpoint mapping enabled us to successfully design primers and obtain the breakpoint sequences in $86 \%$ of the patients (37 out of the 42 patients from group 1 and 2, and 1 patient from group 3) (Table 2). Taking into account that complex rearrangements would have more than one aberrant junction in a single patient and excluding familial cases with similar rearrangements, we expected to find 45 different junction sequences. In all, 33 of them $(73.3 \%)$ were correctly identified: all simple deletions and triplications cases (25/25 and $2 / 2$, respectively), $62.5 \%$ $(5 / 8)$ of simple duplication but only $20 \%$ (2/10) of complex rearrangement junctions. All breakpoints in unrelated patients were unique with no clustering, even in the frequently rearranged introns 2,7 or 44 . Microhomology up to $9 \mathrm{bp}$ was evidenced in $60.6 \%$ of the preserved ends of the rearrangement breakpoints (20 cases out of 33). In nine other cases, insertions up to $25 \mathrm{bp}$ represented mostly the small duplicated parts of sequences surrounding the junctions and 


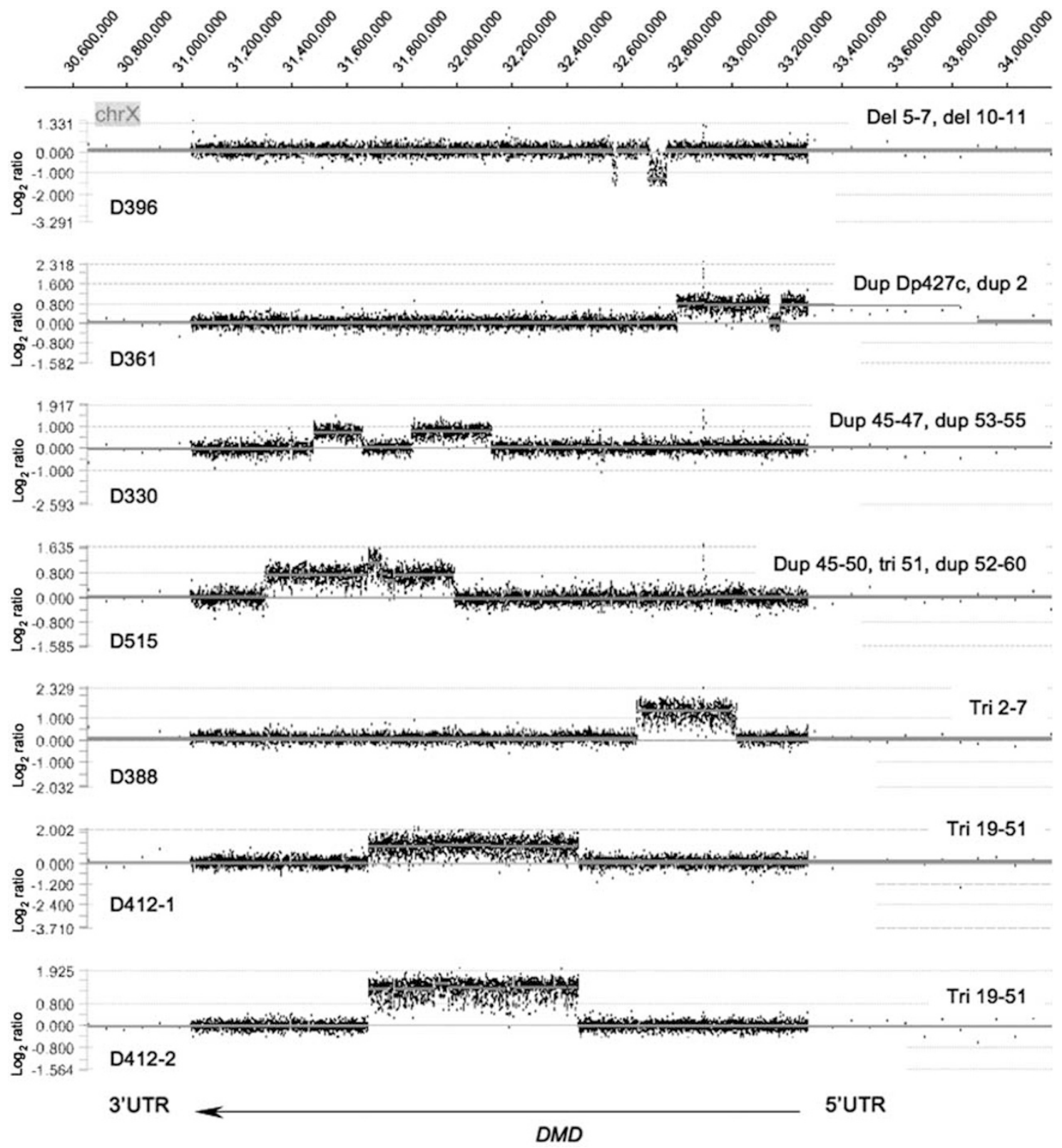

Figure 1 Array-CGH results in patients with complex rearrangements in the $D M D$ gene. Array-CGH $\log _{2}$-ratio profiles of patients with $D M D$ complex rearrangements analyzed with segMNT algorithm by NimbleScan ver.2.5 and displayed on SignalMap ver.1.9 software (Roche NimbleGen, Inc.): data for the signal of each probe were plotted indicating gain or loss of material on the $y$-axis versus $\mathrm{X}$-chromosomal position of the probes on the $\mathrm{x}$-axis accordingly to the GenBank NC_000023.9 and the Human Genome reference sequence Mar.2006 NCBI Build 36.1/hg18 (http://genome.ucsc.edu/). The DMD gene coordinates on the X-chromosome are indicated at the top (RefSeq NC_000023.9), with exon 1 to 79 from right to left. 5'UTR/3'UTR, DMD 5'/3' untranslated regions; del, deletion; dup, duplication; tri, triplication; involved exons are indicated.

only four cases did not show any homology. Overall, repetitive sequences of different classes, such as LINE, LTR, SINE and DNA, were represented in 32 out of 66 junction ends (48.5\%), but there was a marked difference of their involvement in aberrations of exons 3 to $7(64.3 \%$; 9/14) compared with mutations in the major hot spot $(39.3 \% ; 11 / 28)$. No extensive homology was visible even when repetitive elements met on the both sides of the junction with one exception: $90 \%$ homology of about 400 bp of two LINE:L1 elements situated on complementary strands was noted in a distance of $360 \mathrm{bp}$ and $535 \mathrm{bp}$ from the proximal and distal ends of the exons 48-50 deletion junction (D55), respectively (Supplementary Figure 1).
Five out-of-frame deletions of exons 3 to 7 were associated with BMD phenotype but no specific molecular features were found to explain the phenotype/genotype discrepancy in these patients. On the other hand, the two out-of-frame duplications of exons 3 to 7 were confirmed to be in tandem and corresponded to the severe DMD phenotype. Among the nine other cases with exception to the reading-frame rule (5 DMD, 1 symptomatic female and 3 BMD), our findings brought the explanation of severe DMD phenotype in one patient (D145) carrying an in-frame deletion of exons 35 to 42 . Sequencing across the junction revealed a complex pattern on genomic level with putative splice sites in a suitable position to explain the 


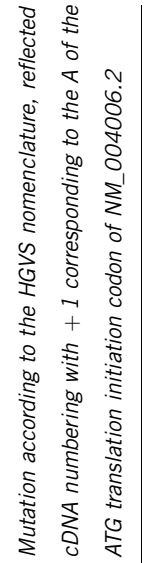

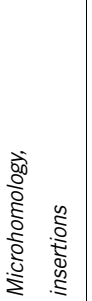

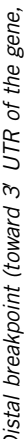

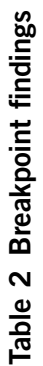

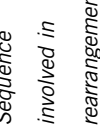

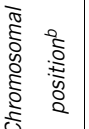

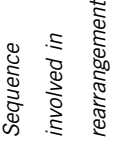

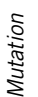
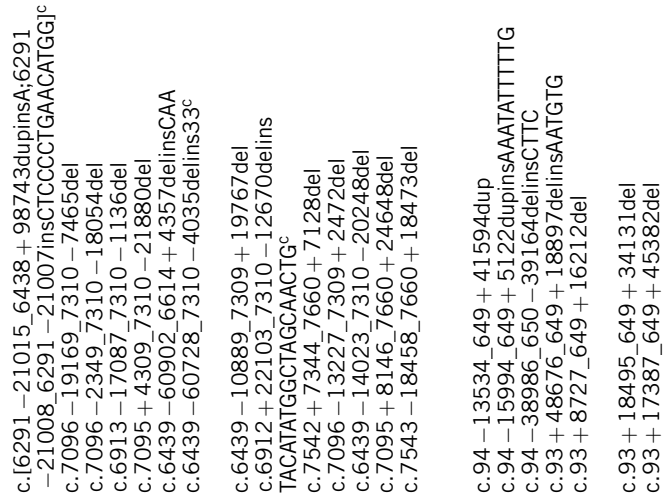

बंगे बु

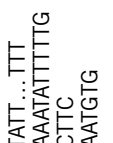

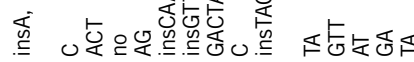

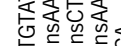

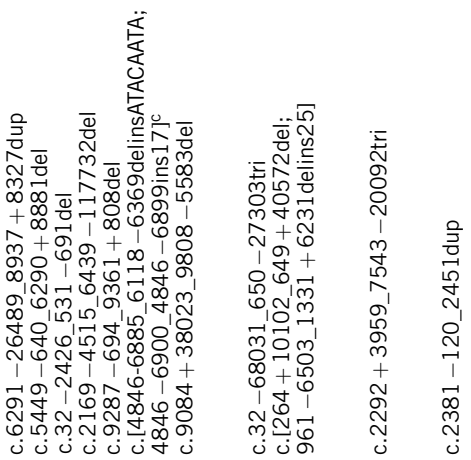

紊

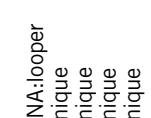

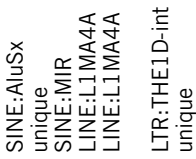

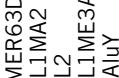

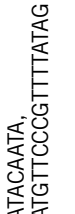

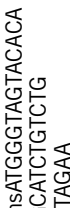

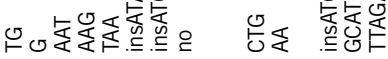

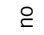

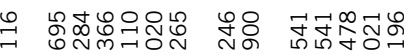

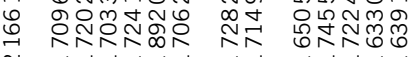

ले $\vec{m} \vec{m} \vec{m} \vec{m} \vec{m} \vec{m} \vec{m} \vec{m} \vec{m} \vec{m}$

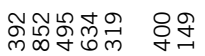

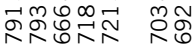

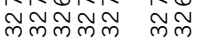

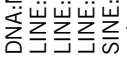

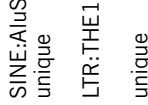

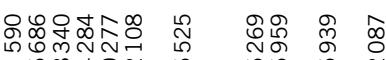

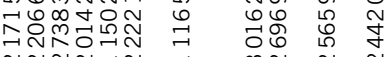

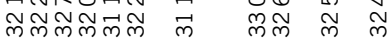

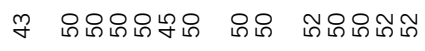

nNAN

mฺฺ

$\neg \curvearrowright \exists \stackrel{\infty}{=}$

$\stackrel{9}{-}$

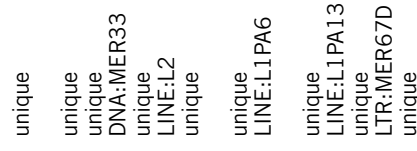

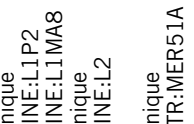

这

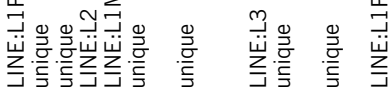

స్

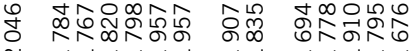

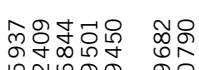

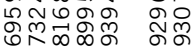

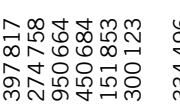

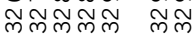

$\vec{m} \tilde{m} \tilde{m} \vec{m} \tilde{m} \vec{m}$

गुनำ

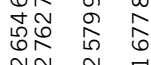

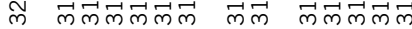

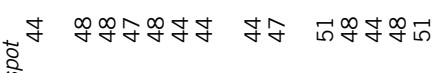

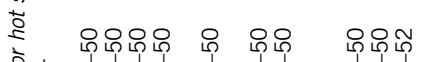

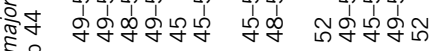

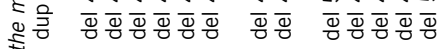

d nnnNa na

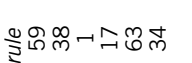

ल) $\tilde{m} \vec{m}$

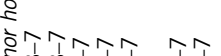

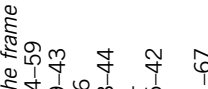

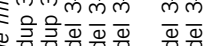

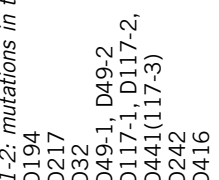

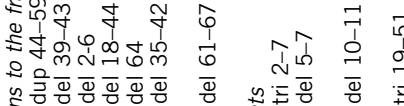

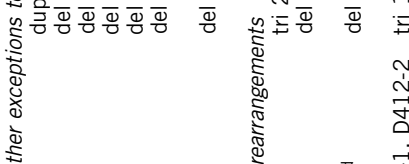

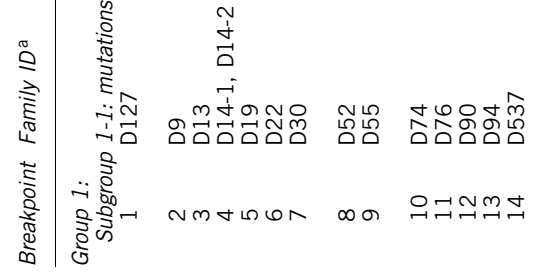

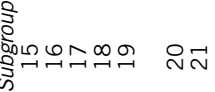

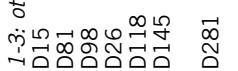

$\times \infty 0$ i

हो.

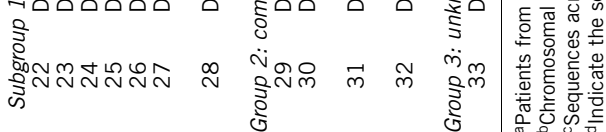


167-bp inclusion in the mature transcripts between exons 34 and 43 detected several years ago in the patient, and of unknown origin at that time (Supplementary Figure 2). Another example of tandem out-offrame duplication of exon 44 (D127) also held a compound breakpoint junction but in this case the 58-bp pseudoexon sequence inserted between the two duplicated copies of exon 44 on the transcripts, was originated from a DNA:MER1A element in intron 43 in a distance of $3 \mathrm{~kb}$ from the aberrant duplication junction (data not shown).

\section{DISCUSSION}

In this study, we present the advent of a high-resolution customdesigned oligonucleotide array-CGH into clinical practice of a reference diagnostic laboratory for DMD. This method showed to be accurate and highly sensitive, cost-effective (price $75-100 €$ per one patient per one experiment for reagents and consumables) and able to detect rearrangements, which are different in type, size and localization in a time less than 5 days for one experiment of 12 patients simultaneously. Based on our practice guidelines for molecular diagnosis of $\mathrm{DMD}$, we recommend that the results have to be supported with alternative diagnostic methods.

With our design, we achieved a very high resolution of array-CGH $(<0.2 \mathrm{~kb})$ both in males and females and in one case of prenatal diagnosis. We noticed that rare experimental imperfections around particular DMD regions (exons 13, 17, 45, Dp140, Dp71 and intron 67) might be conditioned by poor hybridization or, in contrast, partial cross-hybridization of the probes. This fact was conclusive that the design of the probes is determinant for the reliability of the results and gives us the clue for the future probe redesigning.

Despite the high incidence of detected alterations in the DMD gene, ${ }^{5-7}$ little is known about their causative molecular mechanisms. In our study, microhomology was present in $60.6 \%$ of breakpoints being comparable to the findings of Mitsui et al $(2010)^{5}$ for the DMD gene. There were no breakpoint clustering noticed and different families of known repetitive sequences, whose role has already been demonstrated $^{8,9}$ in other diseases, were found in $48.5 \%$ of the junctions. However, this frequency does not differ significantly from that of transposable repetitive elements in the human genome $(46 \%)^{9}$ and could explain our findings. Finally, no low-copy repeats with extensive homology that could participate in DNA secondary structure formation was evidenced except in the $120-\mathrm{kb}$ deletion junction involving exons 48-50. These observations supports the microhomology mediated mechanism model, which could be either non-homologous end-joining (NHEJ) or any alternative replicationbased processes, ${ }^{8}$ in the occurrence of $D M D$ rearrangements.

Although all the deletion breakpoints were obtained, the sequence of only five duplication and two triplication breakpoints were acquired, confirming the hypothesis of tandem ('head-to-tail') junction. Six cases including three duplications, two double duplications and a triplication built in the duplication remained undetected on the sequence level. Because array-CGH gives only information about size of copy number gains and losses, but not their exact position and orientation, we anticipated the difficulties to obtain the duplication/ triplication breakpoints due to their unknown genomic configuration and possibility of aberrant sequence insertions inside the junctions. The absence of amplification with different combinations of primers indirectly tends to confirm this hypothesis.

In conclusion, this large survey of 50 patients confirmed the previous observations ${ }^{10-13}$ that array-CGH is a reliable and effective tool in detecting simple and complex DMD rearrangements. This approach offers some advantages over exon-based detection methods as it can identify pure intronic pathogenic events and it allows precise delineation of rearrangements, some of which may affect the splicing process. This is of high importance for the deep family investigation and a more accurate genotype/phenotype correlations, but also might be decisive factor for the optimal inclusion of patients in clinical trials. In general, it could lead to better understanding of the common fundamental mutational mechanisms, clarifying pathogenesis of diseases associated with instability in the genome.

\section{CONFLICT OF INTEREST}

The authors declare no conflict of interest.

\section{ACKNOWLEDGEMENTS}

This work was supported by an Association Française contre les Myopathies (AFM), grant (AFM-14178 and AFM-15258) to AI. We thank all the physicians, in particular Pr P Sarda, Dr U Walther-Louvier, Dr E Campana Salort, Dr R Bernard, Dr L Lazaro, Dr S Mercier, Dr M Cossée, Dr A David, Pr B Leheup, Dr X Ferrer, Pr PS Jouk, Pr Ph Labrune for providing clinical information and patient material. We are grateful to Céline Saquet and Sylvie Chambert for DNA and RNA analyses in some of the patients, and Nicolas Gilbert for helpful discussion about repetitive elements.

1 Tuffery-Giraud S, Beroud C, Leturcq F et al: Genotype-phenotype analysis in 2,405 patients with a dystrophinopathy using the UMD-DMD database: a model of nationwide knowledgebase. Hum Mutat 2009; 30: 934-945

2 Abbs S, Tuffery-Giraud S, Bakker E, Ferlini A, Sejersen T, Mueller CR: Best practice guidelines on molecular diagnostics in Duchenne/Becker muscular dystrophies. Neuromuscul Disord 2010; 20: 422-427.

3 Faivre L, Khau Van Kien P, Callier P et al: De novo 15q21.1q21.2 deletion identified through FBN1 MLPA and refined by $244 \mathrm{~K}$ array-CGH in a female teenager with incomplete Marfan syndrome. Eur J Med Genet 2010; 53: 208-212.

4 Wildeman M, van Ophuizen E, den Dunnen JT, Taschner PE: Improving sequence variant descriptions in mutation databases and literature using the Mutalyzer sequence variation nomenclature checker. Hum Mutat 2008; 29: 6-13.

5 Mitsui J, Takahashi Y, Goto J et al: Mechanisms of genomic instabilities underlying two common fragile-site-associated loci, PARK2 and DMD, in germ cell and cancer cell lines. Am J Hum Genet 2010; 87: 75-89.

6 Oshima J, Magner DB, Lee JA et al: Regional genomic instability predisposes to complex dystrophin gene rearrangements. Hum Genet 2009; 126: 411-423.

7 Toffolatti L, Cardazzo B, Nobile C et al: Investigating the mechanism of chromosomal deletion: characterization of 39 deletion breakpoints in introns 47 and 48 of the human dystrophin gene. Genomics 2002; 80: 523-530.

8 Chen JM, Cooper DN, Ferec C, Kehrer-Sawatzki H, Patrinos GP: Genomic rearrange ments in inherited disease and cancer. Semin Cancer Biol 2010; 20: 222-233.

9 Belancio VP, Deininger PL, Roy-Engel AM: LINE dancing in the human genome: transposable elements and disease. Genome Med 2009; 1: 97.

10 Bovolenta M, Neri M, Fini S et al: A novel custom high density-comparative genomic hybridization array detects common rearrangements as well as deep intronic mutations in dystrophinopathies. BMC Genomics 2008; 9: 572.

11 del Gaudio D, Yang Y, Boggs BA et al: Molecular diagnosis of Duchenne/Becker muscular dystrophy: enhanced detection of dystrophin gene rearrangements by oligonucleotide array-comparative genomic hybridization. Hum Mutat 2008; 29: 1100-1107.

12 Hegde MR, Chin EL, Mulle JG, Okou DT, Warren ST, Zwick ME: Microarray-based mutation detection in the dystrophin gene. Hum Mutat 2008; 29: 1091-1099.

13 Saillour Y, Cossee M, Leturcq F et al: Detection of exonic copy-number changes using a highly efficient oligonucleotide-based comparative genomic hybridization-array method. Hum Mutat 2008; 29: 1083-1090.

14 Tuffery-Giraud S, Saquet C, Chambert S et al: The role of muscle biopsy in analysis of the dystrophin gene in Duchenne muscular dystrophy: experience of a national referral centre. Neuromuscul Disord 2004; 14: 650-658.

15 Rivier F, Tuffery S, Jellali AJ, Echenne B, Mornet D, Pons F: Mosaic expression of two dystrophins in a boy with progressive muscular dystrophy. Muscle Nerve 1998; 21: 1317-1320.

16 Desmet FO, Hamroun D, Lalande M, Collod-Beroud G, Claustres M, Beroud C: Human Splicing Finder: an online bioinformatics tool to predict splicing signals. Nucleic Acids Res 2009; 37: e67. 